\title{
Establishing an Adult Mouse Brain Hippocampal Organotypic Slice Culture System that Allows for Tracing and Pharmacological Manipulation of ex vivo Neurogenesis
}

Steffen Mayer ${ }^{1,2, *}$ and Charles ffrench-Constant ${ }^{1}$

\author{
${ }^{1}$ MRC Centre for Regenerative Medicine, University of Edinburgh, Edinburgh, United Kingdom; \\ ${ }^{2}$ University Hospital Essen, Department of Endocrinology, University of Duisburg-Essen, Essen, \\ Germany \\ *For correspondence: steffen.mayerl@uk-essen.de
}

[Abstract] The function of the hippocampus depends on the process of adult hippocampal neurogenesis which underpins the exceptional neural plasticity of this structure, and is also frequently affected in CNS pathologies. Thus, manipulation of this process represents an important therapeutic goal. To identify potential strategies, organotypic adult brain slices are emerging as a valuable tool. Over the recent years, this methodology has been refined and here we present a combined protocol that brings together these refinements to enable long-term culture of adult hippocampal slices. We employ a sectioning technique that retains essential afferent inputs onto the hippocampus as well as serum-free culture conditions, so allowing an extended culture period. To sustain the neurogenic potential in the slices, we utilize the gliogenesis-inhibitor Indomethacin. Using EdU retention analysis enables us to assess the effects of pharmacological intervention on neurogenesis. With these improvements, we have established an easy and reliable method to study the effects of small molecules/drugs on proliferation and neuron formation ex vivo which will facilitate future discovery driven drug screenings.

Keywords: Adult hippocampal neurogenesis, Organotypic adult slice culture, Drug screening, Indomethacin, Thyroid hormone, Mct8

[Background] The hippocampus is a unique region of the brain with a high degree of plasticity as a result of the ongoing neurogenesis in the dentate gyrus throughout life. This process of adult hippocampal neurogenesis starts with the asymmetric division of neural stem cells (NSCs) in the subgranular zone (SGZ) that preserves the stem cell pool and generates progenitor cells poised for neuronal differentiation (Kempermann et al., 2004; Anacker and Hen, 2017; Toda and Gage, 2018). These latter cells go through a well-defined sequence of distinct stages: NSC-generated transiently amplifying progenitor cells divide rapidly and give rise to neuroblasts. Neuroblasts are characterized by their expression of the immature neuronal marker Doublecortin (Dcx). They exit the cell cycle to differentiate into immature post-mitotic neurons that in addition to Dcx transiently express the Calcium-binding protein Calretinin as well as the neuronal marker NeuN. In the final step, these newly generated neurons migrate and integrate functionally into the existing neuronal network as mature granule cell neurons which cease to express Dcx and Calretinin, but maintain NeuN expression.

Adult hippocampal neurogenesis plays a fundamental role in physiological CNS functions such as 
Please cite this article as: Mayerl and ffrench-Constant, (2021). Establishing an Adult Mouse Brain Hippocampal Organotypic Slice Culture System that

memory consolidation and cognitive flexibility. Thus, not surprisingly, it is implicated in pathologies like Alzheimer's disease, depression, or schizophrenia (Winner and Winkler, 2015; Anacker and Hen, 2017; Moreno-Jimenez et al., 2019; Park, 2019) and therefore represents an important target for pharmaceutical intervention. However, drug testing is currently often based on cell culture systems that lack the complex architecture and connectivity of the intact brain (Pena, 2010; Humpel, 2015). On the other hand, drug testing in the intact brains of hundreds of animals is not practicable. Organotypic brain slices thus constitute a potential intermediate step as they maintain higher brain cyto-architecture and, as multiple sections can be obtained from one brain, enable comparison of different time points and/or drug concentrations with greatly reduced inter-animal variability. To study the effects of these drugs on neurogenesis, however, a reliable and easy lineage tracing approach is required.

Previously, Kim and colleagues established a protocol to culture adult mouse organotypic hippocampal slices for an extended period of four weeks by employing a serum-free culture medium (Kim et al., 2013). Moreover, they introduced a Hibernate-A based dissection medium as an alternative to more complicated media that required constant gassing with $\mathrm{CO}_{2}$. However, they were unable to trace the fate of single cells, nor did their slices maintain the essential afferent connection to the entorhinal cortex. Kleine Borgmann and co-workers used a different sectioning approach to overcome the latter problem and employed retrovirus labelling to study lineage progression in the SGZ (Kleine Borgmann et al., 2013). Here, we combined and refined these existing organotypic adult slice culture protocols. Moreover, we established EdU label tracing as a suitable read-out for neurogenesis. To circumvent the problem of a decreased neurogenic efficiency following a prolonged culture period, we utilized the Cyclooxygenase-inhibitor Indomethacin that has been shown before to reduce gliogenesis and exert protective effects on neurogenesis in vivo and ex vivo (Gerlach et al., 2016; Hain et al., 2018; Melo-Salas et al., 2018). Finally, establishing proof of concept as to the value of this protocol we compared the effects of Silychristin, iopanoic acid, and $\mathrm{BCH}$ on neurogenesis. These drugs are small molecular inhibitors of the monocarboxylate transporter 8 (Mct8), deiodinase type 2 (Dio2), and L-type amino acid transporters 1/2 (Lat 1/2) respectively-components of central thyroid hormone signalling. While neither iopanoic acid nor $\mathrm{BCH}$ treatment altered ex vivo neurogenesis, the number of new neurons was significantly reduced in Silychristin-treated cultures (Mayerl et al., 2020).

Taken together, these improvements enabled us to establish a method that allows for i) an extended culture period of adult brain slices for at least three weeks with good neurogenic efficiency, ii) easy lineage tracing using EdU that also provides a defined and controlled starting point, and iii) pharmacological manipulation of ex vivo hippocampal neurogenesis. We believe that our advanced methodology will be useful for future drug screening approaches to sustain or improve hippocampal neurogenesis in various pathological conditions or physiological alterations such as ageing.

\section{Materials and Reagents}

1. $10 \mathrm{~cm}$ Petri dish (Thermo Fisher Scientific, Corning, catalog number: BP94A01)

2. 6-well-plate (Corning, catalog number: 3516) 
3. 12-well-plate (Corning, catalog number: 3513)

4. Tin foil

5. Microscope slides SuperFrost Plus ${ }^{\mathrm{TM}}$ (Thermo Fisher Scientific, catalog number: 10149870)

6. Coverslips (e.g., $24 \times 50 \mathrm{~mm}$ ) (Thermo Scientific Menzel x1000 Coverslip $24 \times 50 \mathrm{~mm} \# 1$, catalog number: 15737592)

7. Peel-A-Way ${ }^{\circledR} 22 \times 40 \mathrm{~mm} \times 20 \mathrm{~mm}$ deep, rectangular embedding molds (Ted Pella, catalog number: 27114)

8. Millicell Cell Culture Insert, $30 \mathrm{~mm}$, hydrophilic PTFE, $0.4 \mu \mathrm{m}$ pore size (Merck, catalog number: PICMORG50), shelf storage

9. Mice (e.g., C57/BI6N)

10. EdU (5-ethynyl-2-deoxyuridine) (Thermo Fisher Scientific, Invitrogen ${ }^{\mathrm{TM}}$, catalog number: A10044 (50 mg), stored in $500 \mu$ aliquots at $-20^{\circ} \mathrm{C}$

11. Hibernate ${ }^{\mathrm{TM}}-\mathrm{A}$ Medium (Thermo Fisher Scientific, Gibco ${ }^{\mathrm{TM}}$, catalog number: $\mathrm{A} 1247501$ ), stored at $4{ }^{\circ} \mathrm{C}$ for a maximum of 4 months

12. B-27 $7^{\mathrm{TM}}$ supplement (50x) (Thermo Fisher Scientific, Gibco ${ }^{\mathrm{TM}}$, catalog number: 17504001), aliquots stored at $-20^{\circ} \mathrm{C}$, after defrosting stored at $4{ }^{\circ} \mathrm{C}$ for a maximum of 1 month

13. Agarose, low melting point (Merck, Calbiochem, catalog number: $2070-100 \mathrm{GM}$ ). $4 \%$ solution in PBS can be stored on shelf for up to 6 months and re-used 3-4x

14. Neurobasal ${ }^{\mathrm{TM}}-\mathrm{A}$ Medium (Thermo Fisher Scientific, Gibco ${ }^{\mathrm{TM}}$, catalog number: 10888022), stored at $4{ }^{\circ} \mathrm{C}$ for a maximum of 4 months

15. Indomethacin (Merck, Sigma-Aldrich, catalog number: $17378-5 \mathrm{G}$ ), stored at $4{ }^{\circ} \mathrm{C}$ as stock solution of $100 \mathrm{mM}$ in DMSO for a maximum of 4 months

16. Silychristin (Merck, Sigma-Aldrich, catalog number: $51681-10 \mathrm{MG}$ ), stored at $4{ }^{\circ} \mathrm{C}$ as a stock solution of $100 \mathrm{mM}$ in DMSO for a maximum of 4 months

17. Iopanoic acid (Merck, Sigma-Aldrich, catalog number: 10330000), stored at $4{ }^{\circ} \mathrm{C}$ as a stock solution of $100 \mathrm{mM}$ in DMSO for a maximum of 4 months

18. $\mathrm{BCH}$ (R\&D Systems, catalog number: $5027 / 50$ ), stored at $4{ }^{\circ} \mathrm{C}$ as a stock solution of $100 \mathrm{mM}$ in Neurobasal A for a maximum of 4 months

19. Formaldehyde 37\% (Merck, Sigma-Aldrich, catalog number: 252549-500ML), stored in a ventilated fume hood

20. Click-iT ${ }^{\circledR}$ EdU Alexa Fluor ${ }^{\circledR} 647$ Imaging Kit (Thermo Fisher Scientific, Molecular Probes, catalog number: C10340), components diluted and stored according to manufacturer's instructions

21. Rabbit-anti-Ki67 antibody (Abcam, catalog number: ab16667), aliquots stored at $-20{ }^{\circ} \mathrm{C}$

22. Guinea pig-anti-Doublecortin (Dcx) antibody (Merck, Millipore, catalog number: ab2253), aliquots stored at $-20^{\circ} \mathrm{C}$

23. Mouse-anti-NeuN antibody (Merck, Millipore, catalog number: mab377), stored at $4{ }^{\circ} \mathrm{C}$

24. Fluoromount- $\mathrm{G}^{\circledR}$ (Southern Biotech, catalog number: 0100-01), stored at room temperature

25. Super glue 
26. Triton X-100 (Merck, Sigma-Aldrich, catalog number: X100-1L), stored at room temperature

27. Glycine (Merck, Sigma-Aldrich, catalog number: G7126-1KG), stored at room temperature

28. 10x PBS (Thermo Fisher Scientific, Life Technologies, catalog number: 70011-036 500ml), stored at room temperature; dilute with distilled water

29. BSA (Merck, Sigma-Aldrich, catalog number: A7906-50G), stored at $4{ }^{\circ} \mathrm{C}$

30. Goat serum (Merck, Sigma-Aldrich, catalog number: G6767-100ML), stored at $-20^{\circ} \mathrm{C}$

31. Hoechst33258 (Thermo Fisher Scientific, Life Technologies, catalog number: 62249 5ML), stored at $4{ }^{\circ} \mathrm{C}$

32. L-Glutamine (Invitrogen, catalog number: $25030-024$ ), aliquots stored at $-20{ }^{\circ} \mathrm{C}$

33. Penicillin/Streptomycin $(10,000 \mathrm{U} / \mathrm{ml}$ Pen; $10,000 \mu \mathrm{g} / \mathrm{ml}$ Strep; Invitrogen, catalog number: 15140-122), aliquots stored at $-20^{\circ} \mathrm{C}$

34. DMSO (Thermo Fisher Scientific, catalog number: D12345 3ML), stored at room temperature

35. $7 \mathrm{ml}$ tubes (Sarstedt, Tube $7 \mathrm{ml} 47 \times 20 \mathrm{PC}+$ Cap, catalog number: 71.9923 .610 ), stored at room temperature

36. Dissection buffer (see Recipes)

37. Serum-free slice culture medium (see Recipes)

\section{Equipment}

1. Pipettes

2. Brush

3. Spatula (Thermo Fisher Scientific, Fisherbrand ${ }^{\mathrm{TM}}$ Nickel Chattaway Spatula, catalog number: 11583462)

4. Scalpel (disposable scalpel, Swann Morton No. 11, Medisave, catalog number: SKU0503)

5. Tissue culture hood

6. Water bath

7. Forceps (Fine Science Tools, Dumont \#5 Forceps Standard Carbon, catalog number: 11251-10)

8. Scissors (Fine Science Tools, Fine Scissors, Sharp, catalog number: 14060-10)

9. Rocker

10. Confocal microscope (Leica SP8)

11. Vibratome (Leica VT1000 S)

\section{Software}

1. ImageJ ( $\mathrm{NIH}$, https://imagej. nih.gov/ij/) 


\section{Procedure}

A. Preparation of slices

1. For EdU label tracing studies, inject a 6-8-week old C57/BI6N mouse i.p. with $100 \mu \mathrm{l} \mathrm{EdU} \mathrm{(10}$ $\mathrm{mg} / \mathrm{ml}$ ). To increase number of labeled cells, we injected EdU twice: $4 \mathrm{~h}$ and $2 \mathrm{~h}$ before sacrifice.

2. Prepare $4 \%$ low melting agarose in $1 \times$ PBS and keep it in a water bath at $37^{\circ} \mathrm{C}$ until use.

3. Sacrifice the animal by exposing it to rising concentrations of $\mathrm{CO}_{2}$.

4. Isolate brain rapidly and transfer it into $2 \mathrm{ml}$ serum-free dissection buffer (Kim et al., 2013) in a $7 \mathrm{ml}$ tube on ice. Allow brain to equilibrate for at least $5 \mathrm{~min}$.

5. Prepare for sectioning (Figure 1A): Transfer brain into a $10 \mathrm{~cm}$ Petri dish (Figure 1B), remove cerebellum and brainstem with a scalpel (Figure 1C) and cut along the midline of the brain (Figure 1D). According to the instructions provided in Kleine Borgmann et al. (2013) place hemispheres on the cut surface (Figure 1E) and remove a small part of the cerebral cortex cutting from dorsolateral to ventromedial at an angle of approximately $70^{\circ}$ (Figures 1F, 1G). Flip the hemispheres onto these new cut surfaces and place them into an embedding mold on ice. Gently fill the mold with $4 \%$ low melting agarose and wait till it solidifies (Figure $1 \mathrm{H}$ ). Trim the agarose block using a scalpel and glue it onto a vibratome tissue platform using super glue (Figure 11). Hemispheres can be embedded, glued and cut separately or in parallel. We obtained the best results by cutting them in parallel oriented in a lateral-to-medial direction (i.e., the lateral part facing the vibratome and being cut first).

6. Transfer platform into the vibratome tissue container filled with chilled dissection buffer. To keep the temperature low, cover the container with ice (if possible) or store it on ice if not in use.

7. Cut $300 \mu \mathrm{m}$ thick sections using high frequency (level 7-8) and low speed (level 1-2).

8. Carefully transfer sections containing the hippocampus into a $10 \mathrm{~cm}$ Petri dish containing 4-5 $\mathrm{ml}$ dissection buffer on ice using a brush and spatula (Figure 1J). Note examples of a well cut section with preserved brain cyto-architecture (Figure $1 \mathrm{~K}$ ) and a badly sectioned slice (Figure 1L). We did not trim the sections as we did not find any discernable difference in long term viability between trimming them so that they only contain the hippocampus and entorhinal cortex or culturing the entire sections. 


\section{bĭo-protocol

A
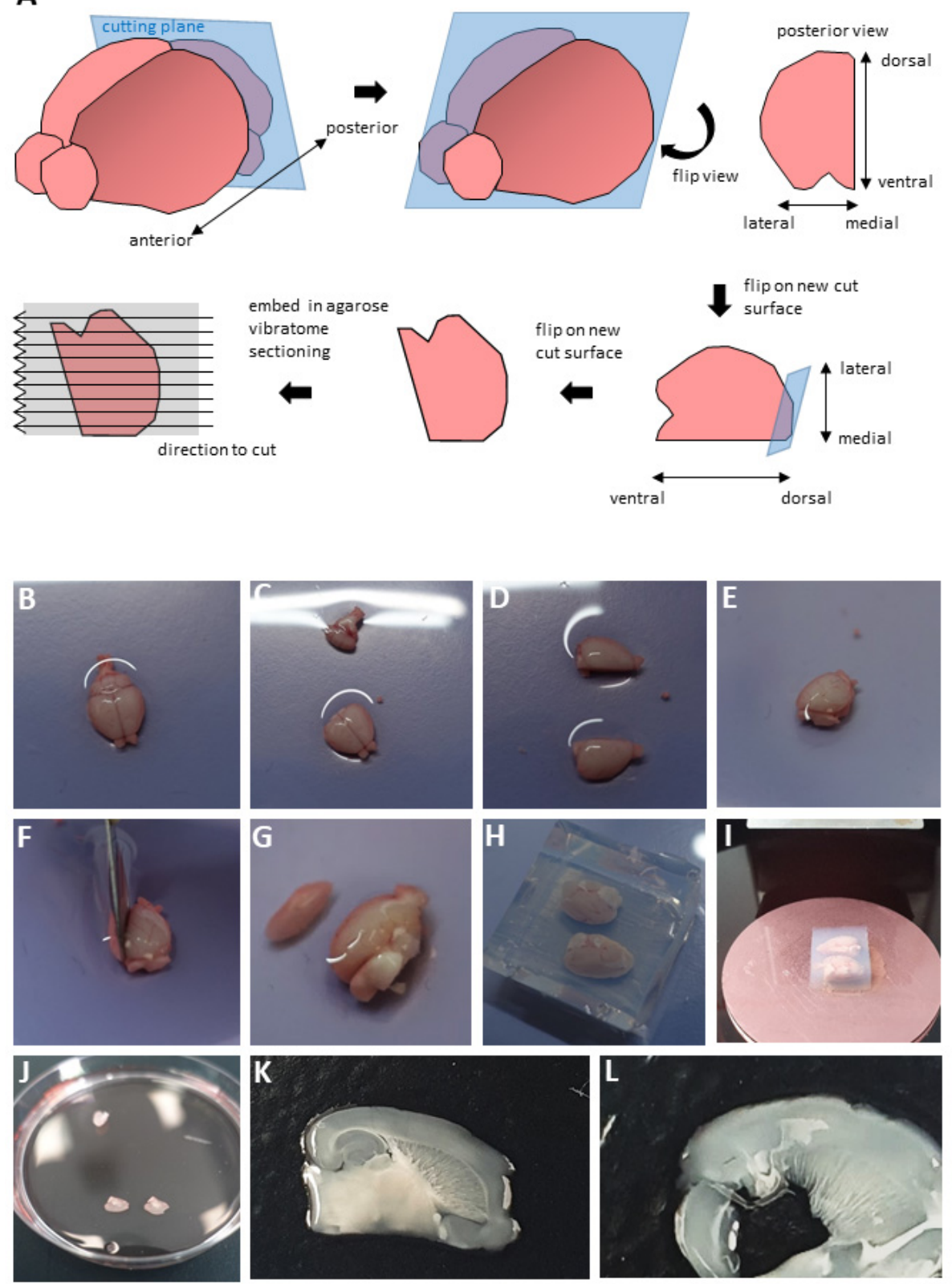

Figure 1. Dissection procedure. (A) Schematic representation of brain sectioning. Panels B-L show photographs of the key steps, as follows. Place the brain in a Petri dish (B). Having cut away cerebellum and brainstem (C), bisect brain along the midline (D). Place hemispheres onto the new cut surfaces $(E)$ and cut off a small part of the cerebral cortex in a dorsolateral to ventromedial direction at an angle of $70^{\circ}(\mathrm{F})$. Flip onto the newest cut surface (G), embed in $4 \%$ low melting agarose $(\mathrm{H})$, glue the block onto a tissue platform (I) and section hemispheres using a vibratome. Collect slices in a $10 \mathrm{~cm}$ Petri dish $(\mathrm{J})$. Representative pictures of a well-cut slice suitable for culturing $(\mathrm{K})$ and of a badly cut slice $(\mathrm{L})$.

\section{B. Slice culture}


1. Before sacrificing the animal, place the required amount of Millicell inserts into 6-well-plate wells.

2. Prepare stock solutions. Dissolve Indomethacin in DMSO to obtain a $100 \mathrm{mM}$ stock solution. Dissolve drugs in DMSO or medium.

3. Add $1 \mathrm{ml}$ serum-free culture medium (Kim et al., 2013) containing Indomethacin and inhibitor (or solvent control) per well. Work in a tissue culture hood. Equilibrate at $37^{\circ} \mathrm{C}$ and $5 \% \mathrm{CO}_{2}$ for one to two hours in a standard cell culture incubator.

4. Add a small drop of culture medium (approx. $50 \mu \mathrm{l}$ ) from the well onto the insert using a standard $200 \mu \mathrm{l}$ pipette to facilitate transfer of the sections. Using a brush and spatula transfer the vibratome sections from chilled dissection medium onto the Millicell inserts so that they are at the air/liquid-interface. Culture 2-3 sections per insert at $37^{\circ} \mathrm{C}$ and $5 \% \mathrm{CO}_{2}$.

5. On the next day, remove the culture medium containing inhibitors or solvent control completely using a $1 \mathrm{ml}$ pipette. Keep the insert in the well and carefully raise it on one side using a pipette tip or forceps to allow for the removal of residual medium. Replace immediately with $1 \mathrm{ml}$ of fresh medium (containing inhibitors) carefully pipetted into the well so that the sections on the insert are once again at the air/liquid interface. Repeat every other day.

\section{Fixation}

1. Fix slices by removing the culture medium and adding $1 \mathrm{ml} 3.7 \%$ formaldehyde in PBS into the well and an additional $1 \mathrm{ml}$ carefully on top of the slices. Fix for $1 \mathrm{~h}$ at ambient temperature.

2. Wash slices $3 \times 20$ min in PBS.

3. Store the fixed slices in PBS at $4{ }^{\circ} \mathrm{C}$ or proceed with immuno-histochemistry.

Note: Slices may detach from the membrane during fixation. This does not affect further steps.

D. Immuno-histochemistry

1. Use forceps, scissors or a scalpel to cut the Millicell insert membrane from the plastic ring.

2. For EdU detection, transfer all slices from the same animal and condition (on the membrane or free-floating) into a 12-well-plate well and permeabilize them in PBS containing $0.1 \%$ Triton X100/0.1 M glycine. Block slices for $2 \mathrm{~h}$ in PBS containg 3\% BSA. Incubate slices with $300 \mu \mathrm{l}$ Click-iT reaction cocktail prepared according to the manufacturer's instructions (Thermo Fisher Scientific Click-i $\mathrm{T}^{\circledR}$ EdU Alexa Fluor ${ }^{\circledR} 647$ Imaging Kit, chapter EdU detection) at room temperature on a rocker for $3 \mathrm{~h}$. For this and all subsequent steps protect the plate from light (e.g., by covering it with tin foil).

3. Block slices in $10 \%$ normal goat serum in PBS containing $0.5 \%$ Triton $\mathrm{X} 100$ overnight at $4{ }^{\circ} \mathrm{C}$.

4. Prepare primary antibodies in blocking buffer $\left(400 \mu \mathrm{l}\right.$ per well). Incubate slices at $4{ }^{\circ} \mathrm{C}$ on a rocker for $48 \mathrm{~h}$. In our experiments, we used rabbit-anti Ki67 (1:250) to visualize proliferating cells, mouse-anti-NeuN (1:300) as a marker for neurons, and guinea pig-anti Dcx (1:300) to label neuroblasts and immature neurons

5. Wash slices $3 \times 1 \mathrm{~h}$ in PBS. 
6. Incubate slices with secondary antibodies (1:500 in blocking buffer) on a rocker at $4{ }^{\circ} \mathrm{C}$ over night.

7. Remove secondary antibodies, add $400 \mu \mathrm{l}$ Hoechst33258 (in water; $5 \mu \mathrm{g} / \mathrm{ml}$ ) per well and incubate for $30 \mathrm{~min}$.

8. Wash slices $3 \times 1 \mathrm{~h}$ in PBS.

9. Using forceps, transfer membranes (slices facing upwards) onto microscope slides or, in case of detached slices, use a brush and spatula.

10. Place the slices in the center of the slide. Add $200 \mu$ Fluoromount-G onto the slices and gently apply a coverslip (e.g., $24 \times 50 \mathrm{~mm}$ ). Be careful not to squeeze the slices as they are extremely thick. Dry at $4{ }^{\circ} \mathrm{C}$.

\section{E. Imaging}

Image slices using a confocal microscope. We employed a Leica SP8 confocal microscope and a 20x objective and imaged a 30-50 $\mu \mathrm{m}$ thick stack in the middle of the $300 \mu \mathrm{m}$ thick slice.

\section{Data analysis}

For data analysis, use the freely available software ImageJ/Fiji. Enumerate marker positive cells (Ki67, Dcx, EdU, NeuN) in the SGZ in one picture every $10 \mu \mathrm{m}$ from the z-stack taken and normalize these numbers to the length of the SGZ (measured using ImageJ's segmented line tool) for quantification (Figure 2). For nuclear markers (Ki67, EdU, NeuN), ascertain that there is a clear overlay with Hoechst33258 staining. Average results from all slices that were obtained from the same animal and cultured under the same condition. In our studies, we quantified EdU incorporation into different cell populations at different time points and then determined statistical significance by unpaired two-tailed Student's $t$-test using slices from 4-6 animals per time point and condition. Depending on the focus of the analysis, one-way or two-way ANOVA may be required, e.g., for longitudinal analysis of the same parameter such as proliferative capacity. To view our results, see Mayerl et al. (2020). 


\section{bĭ̈-protocol
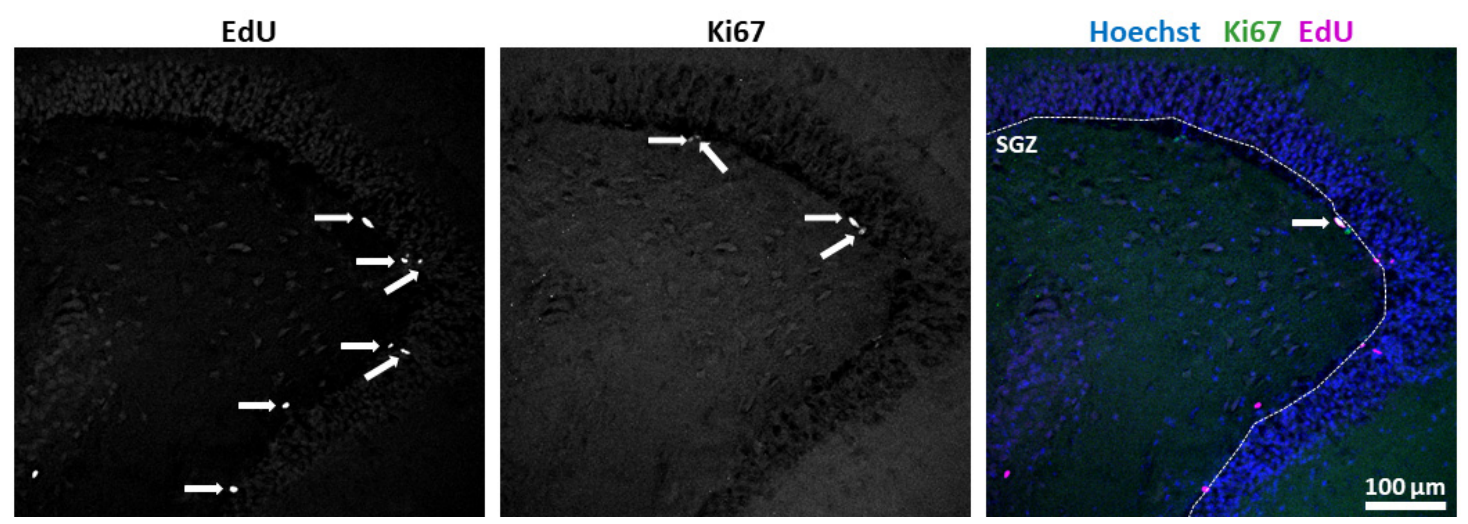

Figure 2. Quantification of results. Marker single or double positive cells along the SGZ were enumerated (arrows). Length of the SGZ was measured using ImageJ's segmented line tool (dotted line). Quotients of positive cell numbers over length were calculated. (scale bar $=100$ $\mu \mathrm{m})$

\section{$\underline{\text { Notes }}$}

1. Note that sacrificing the animal by overexposure to $\mathrm{CO}_{2}$ can be accompanied by a reduction in brain tissue $\mathrm{pH}$ and increase in tissue stiffness (Holtzmann et al., 2016). Whether this change in stiffness alters the quality of the sectioning process in comparison to standard methods of euthanasia such as cervical dislocation or decapitation has not been investigated and may be determined individually.

2. While extracting the brain from the skull be careful not to cut into the brain tissue. Smaller, superficial cuts do not compromise the procedure, but larger incisions will result in cutting problems and ultimately lead to fractured and unsuitable slices. If the latter happens, do not proceed. Likewise, when preparing brain hemispheres for sectioning, take care not to pierce the tissue while trying to hold it in place with forceps. Better to use a blunt spatula to fix the tissue for cutting with the scalpel. When removing a small part of the cortex at a $70^{\circ}$ angle you should be able to perceive a small strip of white matter in the removed part (see Figure 1G). In a few cases, brain hemispheres may not be cut as desired and the slices may be damaged (for comparison see Figures $1 \mathrm{~K}$ and $1 \mathrm{~L}$ ). Problems can include ripped, fractured sections, severing of the hippocampus-cortex connection and visibly unequal thickness over the slice. The latter is common if agarose penetrates underneath a hemisphere while solidifying. As a result, brain tissue will not be glued to the vibratome tissue platform and is free to move if pressure from the blade is applied. If problems occur during cutting our experience is that trouble-shooting possibilities are very limited. We recommend repeating the procedure with another animal instead of using damaged slices.

3. One of the major points that needs considering is the thickness of the slices that-unlike neonatal slices-do not noticeably flatten over the culture period. Thus, the slices produce a pronounced background fluorescent intensity which will be a problem for some antibodies. In 
our hands, well-established, highly specific antibodies worked reliably, while other less specific antibodies did not give staining that could be used for quantifications or did not work at all. Moreover, we usually imaged on the day after mounting. Delayed imaging, however, may lead to the formation of air-filled gaps in the mounting medium that may dry out the sections. Thus, for longer storage before imaging sealing the coverslip e.g. with nail varnish constitutes a feasible option though we never further explored this possibility.

4. We never investigated whether the first block step ( $3 \%$ BSA in PBS) is sufficient and the second block step using $10 \%$ goat serum can be skipped. This likely depends on the performance of the antibodies employed.

5. Moreover, the protocol conditions are optimized for adult stem cells and neurons (hence using Hibernate A, Neurobasal A and Indomethacin) while they might be less efficient in keeping glia cells alive. Therefore, we do not recommend this protocol for the analysis of glia cells unless the culture conditions are further optimized.

\section{Recipes}

Following buffer recipes are based on Kim et al. (2013):

1. Dissection buffer $(100 \mathrm{ml})$

$96 \mathrm{ml}$ Hibernate A

$2 \mathrm{ml} \mathrm{B}-27^{\mathrm{TM}}$ supplement (50x)

$1 \mathrm{ml} 200 \mathrm{mM}$ L-Glutamine

$1 \mathrm{ml}$ Penicillin/Streptomycin

2. Serum-free slice culture medium $(100 \mathrm{ml})$

$96 \mathrm{ml}$ Neurobasal A

$2 \mathrm{ml} \mathrm{B}-27^{\mathrm{TM}}$ supplement (50x)

$1 \mathrm{ml} 200 \mathrm{mM}$ L-Glutamine

$1 \mathrm{ml}$ Penicillin/Streptomycin

$80 \mu 100 \mathrm{mM}$ Indomethacin

\section{Acknowledgments}

This work was supported by a grant from the DFG to SM (MA7212/2-1), by a Wellcome Trust Senior Investigator award (104783/Z/14/Z) and funding from the BBSRC (BB/L00402X/1) to CffC. We thank SCRM animal facility staff (Luke McPhee, Chris Wilson, Lorraine McNeil, John Agnew, and Jamie Kelly) and SCRM imaging facility staff (Bertrand Vernay) for their excellent help. The protocol was first published in our original research paper "Hippocampal Neurogenesis Requires Cell-Autonomous Thyroid Hormone Signaling" in Stem Cell Reports (Mayerl et al., 2020). 


\section{Competing interests}

The authors declare that no conflict of interests exists.

\section{Ethics}

All studies were executed under Project Licence PPL 70/8748, valid from 6 Dec 2015 to 5 Dec 2020, in compliance with UK Home Office regulations and local guidelines by The University of Edinburgh.

\section{$\underline{\text { References }}$}

1. Anacker, C. and Hen, R. (2017). Adult hippocampal neurogenesis and cognitive flexibility linking memory and mood. Nat Rev Neurosci 18(6): 335-346.

2. Gerlach, J., Donkels, C., Münzner, G. and Haas, C. A. (2016). Persistent gliosis interferes with neurogenesis in organotypic hippocampal slice cultures. Front Cell Neurosci 10: 131.

3. Hain, E. G., Sparenberg, M., Rasinska, J., Klein, C., Akyuz, L. and Steiner, B. (2018). Indomethacin promotes survival of new neurons in the adult murine hippocampus accompanied by anti-inflammatory effects following MPTP-induced dopamine depletion. $J$ Neuroinflammation 15(1): 162.

4. Holtzmann, K., Gautier, H. O. B., Christ, A. F., Guck, J., Káradóttir, R. T., and Franze, K. (2016). Brain tissue stiffness is a sensitive marker for acidosis. J Neurosci Methods 271: 50-54.

5. Humpel, C. (2015). Organotypic brain slice cultures: A review. Neuroscience 305: 86-98.

6. Kempermann, G., Jessberger, S., Steiner, B. and Kronenberg, G. (2004). Milestones of neuronal development in the adult hippocampus. Trends Neurosci 27(8): 447-452.

7. Kim, H., Kim, E., Park, M., Lee, E. and Namkoong, K. (2013). Organotypic hippocampal slice culture from the adult mouse brain: a versatile tool for translational neuropsychopharmacology. Prog Neuropsychopharmacol Biol Psychiatry 41: 36-43.

8. Kleine Borgmann, F. B., Bracko, O. and Jessberger, S. (2013). Imaging neurite development of adult-born granule cells. Development 140(13): 2823-2827.

9. Mayerl, S., Heuer, H. and ffrench-Constant, C. (2020). Hippocampal neurogenesis requires cell-autonomous thyroid hormone signaling. Stem Cell Reports 14(5): 845-860.

10. Melo-Salas, M. S., Perez-Dominguez, M. and Zepeda, A. (2018). Systemic inflammation Impairs proliferation of hippocampal type 2 intermediate precursor cells. Cell Mol Neurobiol 38(8): 1517-1528.

11. Moreno-Jimenez, E. P., Flor-Garcia, M., Terreros-Roncal, J., Rabano, A., Cafini, F., Pallas-Bazarra, N., Avila, J. and Llorens-Martin, M. (2019). Adult hippocampal neurogenesis is abundant in neurologically healthy subjects and drops sharply in patients with Alzheimer's disease. Nat Med 25(4): 554-560. 
12. Park, S. C. (2019). Neurogenesis and antidepressant action. Cell Tissue Res 377(1): 95-106.

13. Pena, F. (2010). Organotypic cultures as tool to test long-term effects of chemicals on the nervous system. Curr Med Chem 17(10): 987-1001.

14. Toda, T. and Gage, F. H. (2018). Review: adult neurogenesis contributes to hippocampal plasticity. Cell Tissue Res 373(3): 693-709.

15. Winner, B. and Winkler, J. (2015). Adult neurogenesis in neurodegenerative diseases. Cold Spring Harb Perspect Biol 7(4): a021287. 